\title{
SOLAR OBSERVATIONS WITH THE LARGE PULKOVO RADIO TELESCOPE AT 3.2-CM WAVELENGTH
}

\author{
v. IKHSANOVA \\ Pulkovo Observatory, Leningrad, U.S.S.R.
}

The observations of the sun by the large Pulkovo radio telescope [1] began in 1956 December. In accordance with the antenna dimensions, a fan-beam diagram about one minute of arc in width and one degree in height was expected (at $\lambda=3.2 \mathrm{~cm}$ ).

The instrument's high resolving power allowed certain identification of local radio sources with sunspot groups [2]. The daily records of the sun during 1956 December 23 to 29 are shown in Fig. 1, which gives the projections of sunspots on an east-west line on December 23 and 29. Vertical lines mark the limits of the solar disk.

One can see that the half-widths of the local regions on the records coincide with those of the aerial diagram; thus, the size of the emitting region does not differ much from one minute of arc.

By taking these dimensions into account, one can find the brightness temperature of the region. This appeared to depend on sunspot-group parameters and to range between 0.3 to $1.0 \times 10^{\circ}{ }^{\circ} \mathrm{K}$. The radio emission of some sources was rather steady for two or three months. It arose with the group, rapidly increased with the

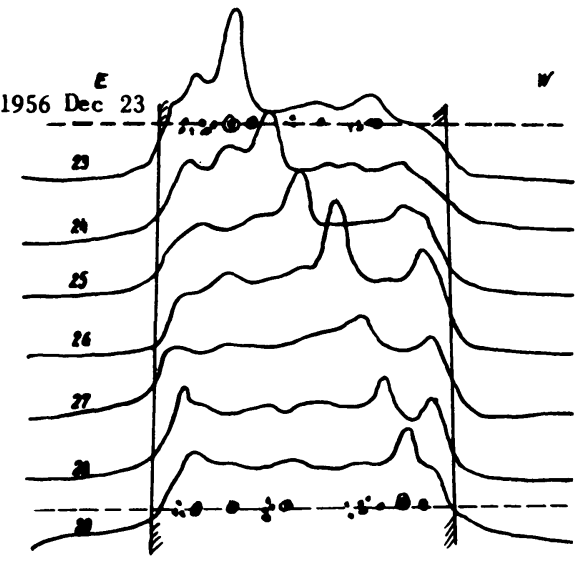

FIG. 1. Daily records. group growth, and remained nearly constant as the group entered class $F$ (Brunner-Waldmeier classification).

Sunspot-group decay was followed by a decrease of radio emission. The radiation disappeared with the sunspots. In some cases the bright green line of the corona was observed in the region where the radio emission had been observed before.

In 1957 and in the beginning of 1958 the motion of the emission regions connected with the sun's rotation was studied. The radio emission regions, situated at some height over the photosphere, were expected to move faster than the corresponding sunspots. Therefore by comparing the distance of a sunspot from the central meridian with that of the emission region the height of the region can be determined. 
Two examples of such height determinations are shown in Fig. 2. The horizontal axis gives sunspot distances from the central meridian; the vertical axis gives those of the radio-emission region. Effective centers of emission appeared to be at the height of $1.07 R_{\odot}$ which is in the lower corona.
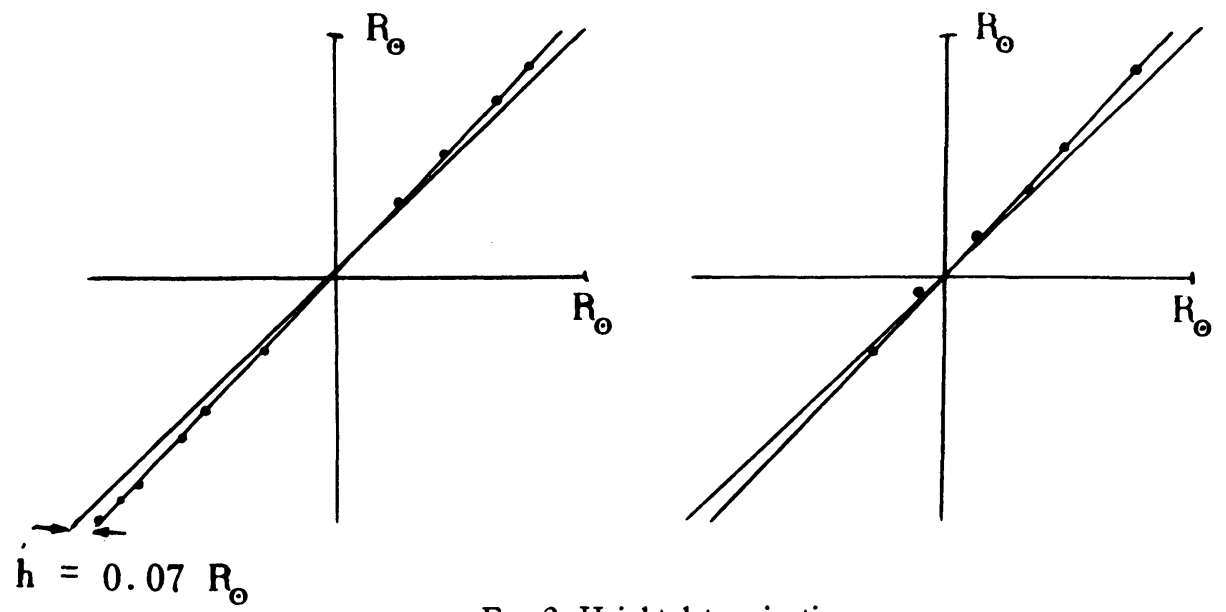

FIG. 2. Height determination.

Of special interest are the regions that appear on the sun's eastern limb one day before the corresponding sunspots and those that can be observed on the sun's western limb one day after the group's disappearance. From studying these regions, it is possible to predict the appearance of sunspot groups and their probable area.

Thus, our radio telescope takes into account the radiation of the local regions connected with the sunspots behind the sun's limb. The correlation between the sunspot area and the solar radiation density was always uncertain owing to the absence of data about these unobserved sunspots. In our case this uncertainty is overcome.

During 1957 July 16 to 30 , U. N. Pariiskii made simultaneous observations of the sun at $\lambda=10 \mathrm{~cm}$ with the same antenna. The distances of the local sources from the central meridian appeared to be the same at both wavelengths.

Since the emission of local regions at $\lambda=3.2 \mathrm{~cm}$ is generated at the height of $1.07 R_{\odot}$ it can be supposed that these regions of microwave emission are located in the corona. This assumption is in agreement with Waldmeier's hypothesis of coronal condensations over sunspots [3].

While at $3.2 \mathrm{~cm}$ wavelength, coronal condensation emission disappears with the corresponding sunspot group, at $7.5 \mathrm{~cm}$ wavelength it could be observed two days longer [4]. This means that the electron density of a coronal condensation is decreasing in such a way that the optical thickness is high enough at $7.5 \mathrm{~cm}$ wavelength to produce the observed radio emission during several days after sunspot decay. At $3.2 \mathrm{~cm}$ wavelength the optical thickness is much less than unity at the same time. Thus the process of dissipation 
of a coronal condensation may be considered in terms of its gradual widening; the process is followed by decreasing radio emission, beginning at the shorter wavelength.

Three components of the thermal solar radio emission can be considered:

1. The first component from the quiet sun can be determined statistically. No change in this level was found (at least during several months of observations). It is shown in Fig. 3. The vertical lines show the limits of the optical disk.

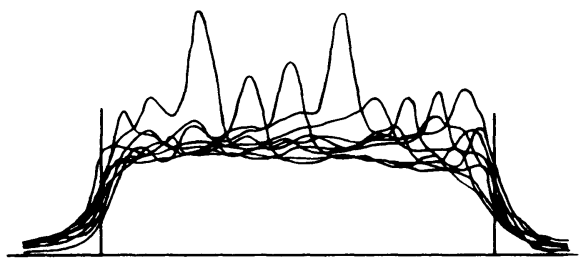

FIG. 3. The basic component.

2. The second component is connected with some active region in the chromosphere. It has an intensity of about 2 per cent of the quiet sun and is of a rather extended area. In some cases this component is correlated with flocculi.

3. The third component is connected with phenomena in the lower corona. It originates in local regions of radio emission and is usually defined as a slowly varying component. The flux from separate coronal condensations ranges between 12 and 20 per cent of the quiet sun's level.

The author is grateful to Professor S. E. Khaikin for advice in this work.

\section{REFERENCES}

[1] Khaikin, S. E., and Kaidanovskii, N. L. Paper 29.

[2] Ikhsanova, V. N. Izv. Obs. Pulkovo, 21, 29, 1958.

[3] Waldmeier, M. Z. Ap. 31, 304, 1953.

[4] Kakinuma, T. Proc. Res. Inst. Atm. Nagoya U. 4, 78, 1956. 